\title{
Should we be concerned about air quality as a contributor to poor outcomes in lung transplant recipients?
}

\author{
Joel D. Kaufman ${ }^{1}$ and Ganesh Raghu²
}

Affiliations: ${ }^{1}$ University of Washington, Depts of Environmental and Occupational Health Sciences, Medicine, and Epidemiology, Seattle, WA, USA. ${ }^{2}$ University of Washington, Depts of Medicine and Laboratory Medicine, Seattle, WA, USA.

Correspondence: Joel D. Kaufman, University of Washington School of Public Health, 1959 NE Pacific St, Box 357230, Seattle, WA 98195-7230, USA. E-mail: joelk@uw.edu

@ERSpublications

Two new papers show adverse effects of air pollution following lung transplantation. What can we conclude? http://ow.ly/Od1k306XIoD

Cite this article as: Kaufman JD, Raghu G. Should we be concerned about air quality as a contributor to poor outcomes in lung transplant recipients? Eur Respir J 2017; 49: 1602369 [https://doi.org/10.1183/ 13993003.02369-2016].

Recurrent and chronic rejection and infection are the principal reasons for poor outcomes in lung transplant recipients who manifest chronic lung allograft dysfunction (CLAD). The aetiology of CLAD is poorly understood and the prognosis is poor [1]. Indeed, environmental factors, including those from work, indoor sources and ambient sources (outdoors) that affect air quality, can be reasonably anticipated to affect the highly susceptible transplanted lungs as much as or more than healthy or diseased native lungs. Hence, there is an interest in studying whether environmental factors influence both the development and progression of post-transplantation complications including CLAD.

The population of lung transplant recipients is diverse, with different underlying diseases, degrees of immune activation and immunosuppression, underlying genetic predisposing factors, comorbid conditions, and microbiological milieu that influence long-term survival and quality of life. Environmental factors, many of which can be investigated and remedied, can include indoor and outdoor air quality as well as biological factors or chemical hazards. Since air pollutants have been shown to have adverse effects on native lungs [2-5], it is entirely plausible that these factors can influence long-term success of allograft lungs. Attention to such factors may provide meaningful nonpharmacological approaches to improving outcomes.

Two articles [6,7] in this issue of the European Respiratory Journal assess the association between ambient air pollution exposures and prognosis following lung transplantation. Taken together, these papers support the hypothesis that living in areas with higher outdoor concentrations of particulate matter air pollution and of traffic-related air pollutants lead to worse outcomes in lung transplant recipients.

In an analysis from the Systems Prediction of Chronic Allograft Dysfunction (SysCLAD) study, SIROUX et al. [6] took advantage of serial lung function studies of 520 lung transplant recipients and found that higher air pollutant levels estimated at the homes of transplant recipients (particulate matter and nitrogen dioxide) were associated with lower vital capacities. RuTTENs et al. [7] analysed CLAD mortality in 5707 patients from 13 European transplant centres, and found increased risk with higher residential estimated particulate matter concentrations and with living near major roads. Interestingly, both showed decreased risk for these effects among the group of transplant recipients taking macrolides. These findings suggest

Received: Dec 022016 | Accepted: Dec 052016

Conflict of interest: None declared.

Copyright @ERS 2017 
that the infectious and/or immunological processes modified by macrolides may also be the active process underlying air pollutant effects.

These studies are both of high quality due to the carefully followed populations, though a number of issues might caution us from drawing a clear causal relationship between pollutants and outcome in lung transplantation. Air quality models, even high-quality ones, provide estimates of outdoor pollutant concentrations that often do not reflect the small-scale variation that exists, especially for traffic-related pollutants; this issue can lead to misclassifying exposures. These two studies are also challenged by the fact that air pollution levels have been declining at the same time that advances in treatment and more widespread use of macrolides have occurred; these temporal trends can make it difficult to disentangle effects. Both papers focus exclusively on outdoor air pollutant concentrations, which are a major source of indoor and individual exposures; the studies did not consider indoor predictors of poor air quality. The SysCLAD analysis is diminished in that it does not address change over time in individuals' FVC, which can be considered a more important metric for understanding the role of an environmental factor in prognosis. Nevertheless, the two studies, along with prior work in this area $[8,9]$, have advanced our understanding and force consideration of how to limit the effects of these environmental factors.

Reducing air pollutant concentrations on a broad population basis is the optimal approach to preventing these effects, there remains great variation in pollution levels between communities, and effects continue to be observed at current concentrations. So, while public health approaches to reducing emissions and improving ambient air quality must push ahead [10,11], these new findings call for attention by those caring for lung transplant recipients. To be specific, management practices for these susceptible patients might consider ways to reduce inhaled exposures to noxious environmental factors.

The studies raise several questions. Are lung transplant recipients more susceptible to environmental insult than other groups? Clearly, this is a group with very high risk of insults to the transplanted lung, but there is not yet definitive information to answer this question. Are outdoor combustion-derived pollutants more important than other types of environmental agents, such as indoor moisture and related biological hazards, that are increasingly of concern as contributors to interstitial lung disease? More research is needed to understand this. How certain do we need to be before making recommendations that impact patient care? Groups recommending care guidelines favour large clinical trials before implementing recommendations, but it can be argued that the preservation of transplanted lungs warrants prudent action to reduce exposures in the setting of consistent observations; informative trials are unlikely to be forthcoming for ethical and financial reasons.

Lung transplant recipients living in more polluted communities should be encouraged to use effective home air purifying filtration devices (with HEPA and activated charcoal elements, not devices which worsen matters by generating ozone). Such filtration devices have been shown to reduce indoor pollutant concentrations and reduce vascular impacts of pollutants [12-16]. Similarly, although less evidence is yet available to support the issue in this specific population, it is also reasonable to identify and remediate indoor causes of poor air quality for this patient group, including poorly ventilated indoor combustion sources and excessive indoor moisture that carries attendant biological hazards. It is more difficult still to make recommendations regarding the wearing of negative pressure filtration masks or avoidance of outside activities altogether in highly polluted locales.

More research is needed to understand the role of specific environmental agents and the effectiveness of exposure reduction in improving prognosis. The post-lung transplantation population represents a group susceptible to effects of environmental factors. Community-level environmental interventions that include adoption and implementation of science-based air quality regulations are the best way to reduce air pollutant effects for everyone with normal and diseased lungs, and perhaps more so for lung transplant recipients. Proactive attention to these patients' environmental exposures as preventive measures and minimise risks for injury to their transplanted lungs is now justified and prudent practice. Society has already made huge investments to enhance survival and improve the quality of life for lung transplant recipients; it is now prudent to pay diligent attention and adhere to the dictum that "prevention is better than cure"

\section{References}

1 Verleden GM, Raghu G, Meyer KC, et al. A new classification system for chronic lung allograft dysfunction. J Heart Lung Transplant 2014; 33: 127-133.

2 Baccarelli A, Kaufman JD. Ambient particulate air pollution, environmental tobacco smoking, and childhood asthma: interactions and biological mechanisms. Am J Respir Crit Care Med 2011; 184: 1325-1327.

3 Goss CH, Newsom SA, Schildcrout JS, et al. Effect of ambient air pollution on pulmonary exacerbations and lung function in cystic fibrosis. Am J Respir Crit Care Med 2004; 169: 816-821.

4 Sack CS, Kaufman JD. Air pollution levels and children's lung health. How low do we need to go? Am J Respir Crit Care Med 2016; 193: 819-820.

5 Young MT, Sandler DP, DeRoo LA, et al. Ambient air pollution exposure and incident adult asthma in a nationwide cohort of U.S. women. Am J Respir Crit Care Med 2014; 190: 914-921. 
6 Siroux V, Benmerad M, Slama R, et al. Chronic effects of air pollution on lung function after lung transplantation in the Systems Prediction of Chronic Lung Allograft Dysfunction (SysCLAD) study. Eur Respir J 2017; 49: 1600206 .

7 Ruttens D, Verleden SE, Bijnens EM, et al. An association of particulate air pollution and traffic exposure with mortality after lung transplantation in Europe. Eur Respir J 2017; 49: 1600484.

8 Bhinder S, Chen H, Sato M, et al. Air Pollution and the development of posttransplant chronic lung allograft dysfunction. Am J Transplant 2014; 14: 2749-2757.

9 Nawrot TS, Vos R, Jacobs L, et al. The impact of traffic air pollution on bronchiolitis obliterans syndrome and mortality after lung transplantation. Thorax 2011; 66: 748-754.

10 Héroux M-E, Anderson HR, Atkinson R, et al. Quantifying the health impacts of ambient air pollutants: recommendations of a WHO/Europe project. Int J Public Health 2015; 60: 619-627.

11 Scale of Europe's air pollution problem demands more action. Lancet 2016; 388: 2714.

12 Allen RW, Carlsten C, Karlen B, et al. An air filter intervention study of endothelial function among healthy adults in a woodsmoke-impacted community. Am J Respir Crit Care Med 2011; 183: 1222-1230.

13 Chen $\mathrm{R}$, Zhao A, Chen $\mathrm{H}$, et al. Cardiopulmonary benefits of reducing indoor particles of outdoor origin: a randomized, double-blind crossover trial of air purifiers. J Am Coll Cardiol 2015; 65: 2279-2287.

14 Giles LV, Barn P, Kunzli N, et al. From good intentions to proven interventions: effectiveness of actions to reduce the health impacts of air pollution. Environ Health Perspect 2011; 119: 29-36.

15 Rajagopalan S, Brook RD. Personalizing your airspace and your health. J Am Coll Cardiol 2015; 65: 2288-2290.

16 Weichenthal S, Mallach G, Kulka R, et al. A randomized double-blind crossover study of indoor air filtration and acute changes in cardiorespiratory health in a First Nations community. Indoor Air 2013; 23: 175-184. 